\title{
Comparison of the Nerve Conduction Parameters in Proximally and Distally Located Muscles Innervated by the Bundles of Median and Ulnar Nerves
}

\author{
Nedim Ongun ${ }^{a}$ Attila Oguzhanoglu ${ }^{b}$ \\ Department of Neurology, ${ }^{a}$ Denizli State Hospital, and ${ }^{b}$ Pamukkale University Hospital, Denizli, Turkey
}

\author{
Key Words \\ Median nerve · Ulnar nerve · Nerve conduction study . \\ Temporal dispersion · Proximal innervation - Distal \\ innervation
}

\begin{abstract}
Objective: The aim of this study was to investigate and compare the conduction parameters of nerve bundles of median and ulnar nerves that innervate proximal and distal muscles. Subjects and Methods: Thirty male and 30 female healthy volunteers between 18 and 70 years of age were enrolled in the study. The conduction parameters were recorded from the proximally located flexor carpi ulnaris, pronator teres and the flexor carpi radialis muscles to the distally located abductor digiti minimi and abductor pollicis brevis muscles for the ulnar and median nerves. Each nerve was stimulated at the region above the elbow and at the axillary region separately. The Student $t$ test was used for statistical analysis, and Levene's test was used to assess whether or not the group variances exhibited a uniform distribution. Results: The conduction velocities were faster $(78.27 \pm 6.55$ vs. $67.83 \pm 6.76 \mathrm{~m} / \mathrm{s}$, and $74.57 \pm 5.66$ and 74.23 $\pm 5.88 \mathrm{vs} .66 .38 \pm 6.85 \mathrm{~m} / \mathrm{s}$ ) and the durations of compound muscle action potential (CMAP) response were longer ( 15.65 \pm 2.43 vs. $13.55 \pm 1.78 \mathrm{~ms}$, and $16.38 \pm 2.39$ and $16.04 \pm 2.34$ vs. $13.40 \pm 1.79 \mathrm{~ms}$ ) in proximally located muscles than in
\end{abstract}

distally located muscles that are innervated either by ulnar or median nerves $(p<0.001)$. However, the CMAP amplitudes were smaller $(2.52 \pm 1.16 \mathrm{vs} .5 .81 \pm 3.13 \mathrm{mV}$, and 2.90 \pm 1.20 and $3.59 \pm 1.66$ vs. $6.88 \pm 2.77 \mathrm{mV}$ ) in proximal muscles than in distal muscles $(p<0.001)$. There was no significant difference $(p>0.05)$ between males and females regarding conduction velocities and CMAP amplitudes recorded from proximal and distal muscles. Conclusion: Proximal muscles innervated by median or ulnar nerves had lower CMAP amplitude values, longer CMAP durations and higher conduction velocities than distal muscles. These findings could reveal a temporal dispersion and phase cancellation due to desynchronized conduction during nerve stimulation.

ㄷ) 2016 S. Karger AG, Basel

\section{Introduction}

The diameter of the peripheral motor nerve gradually decreases until it reaches the target after leaving the spinal cord [1]. In myelinated nerve fibers, the diameter of the nerve fiber is directly proportional to the nerve conduction velocity [1]. Hence, the conduction velocity decreases with the diameter [1]. In contrast, there have also been trials suggesting that there is no difference between the proximal and the distal segment [2]. 
The motor unit size and the nerve fiber diameter correlate with the size of the muscle they innervate $[3,4]$. Therefore, it might be predictable that the nerve fibers of muscles with larger mass and proximal localization are larger and faster [5].

A single peripheral nerve stimulates many muscles and, in the same peripheral nerve, nerve fibers can innervate different muscle ends at different levels. The diameters of the most rapidly conducting fibers differ among the most rapidly conducting nerve fibers innervating a proximal muscle, which has a larger diameter compared to those nerve fibers innervating a distal muscle $[4,6]$. Consequently, if a nerve is stimulated from the same region and an electrophysiological recording is conducted in one proximally and another distally located muscle, data can be obtained about the conduction parameters of the nerve fibers of various diameters [7]. Hence, this study was performed to assess nerve conduction velocities, amplitudes of compound muscle action potential (CMAP) and the durations of a proximal forearm muscle with a relatively larger mass and of thenar muscles that were distally localized with a small mass stimulated by the median nerve. The obtained data were compared to those from the ulnar nerve using the same method and study design.

\section{Subjects and Methods}

The study was approved by the Pamukkale University Non-Interventional Clinical Trials Ethics Committee. Statistical power analysis was performed to estimate the number of subjects needed, and 30 subjects were indicated in minimum. Thirty healthy male subjects and 30 healthy female subjects at the age of between 18 and 70 years with no neuropathy detected via routine electrophysiological protocols, assessed at the electromyography (EMG) laboratory, were included in the study. All of the subjects gave written informed consent and underwent a nerve conduction study in the right upper extremity median nerve and ulnar nerve. The examination was performed with the patient in a supine position, with his or her right arm parallel to his or her trunk and a 180-degree openness in the forearm for median nerve investigation. Data were also collected with the right arm at a 90-degree angle to the trunk and the forearm at a 90-degree angle to the arm for ulnar nerve investigation. Stimuli were applied to the region above the elbow and the axillary region separately. Recordings were also conducted from the proximal flexor carpi ulnaris muscle (FCU) and the distal abductor digit minimi muscle innervated by the ulnar nerve. Recordings were conducted from the proximal pronator teres muscle (PT) and the flexor carpi radialis muscle (FCR) as well as from the distal abductor pollisis brevis muscle innervated by the median nerve.

The study was conducted using a Medelec Synergy Nicolet EDX EMG device (Manor Way, Old Woking, Surrey, UK). The room temperature was maintained between 25 and $28^{\circ} \mathrm{C}$. If re- quired, the extremity was heated. During the trial, the duration of stimulation was set at $100 \mu \mathrm{s}$, the screen sweep time at $40 \mathrm{~ms}$, and the sensitivity was $5 \mathrm{mV}$. 3-Hz low-frequency and $10-\mathrm{kHz}$ highfrequency filters were used.

\section{Stimulation}

The nerves were stimulated using a superficial bipolar electrode with $2-\mathrm{cm}$ constant intervals via supramaximal stimuli. The intensity of the stimulus was slowly increased up to the point at which the CMAP amplitude did not increase any more. Following a $25 \%$ increase in the maximal value of stimulus, this point was determined to be the level of supramaximal stimulation [3].

\section{Ulnar Nerve Stimulation}

On the right arm segment, the point $2 \mathrm{~cm}$ proximal from the medial epicondyle on the sulcus nervi ulnaris on the humerus was selected as the distal stimulus point; in the axillary region, the lateral site of the brachial artery and the medial side of the biceps brachi muscle was selected as the proximal stimulus point [8].

\section{Median Nerve Stimulation}

On the right arm segment, the brachial artery was chosen as the point of brachial artery pulsation, and the medial site of the biceps brachi muscle was selected as the distal stimulus point. In the axillary region, the lateral site of the brachial artery and the medial side of the biceps brachi muscle was selected as the proximal site of stimulation [8].

\section{Recording}

A 50-mm-long superficial bar electrode with a $20 \times 8 \mathrm{~mm}$ recording surface and a $3-\mathrm{cm}$ anode-cathode distance was used during the recording. The active electrode was placed on the middle of the muscle or at the most bellied site while the reference was placed distal to the active electrode.

\section{Muscles}

For FCU, the active electrode was placed a distance of two fingers lateral to the ulna at the junction of the proximal and the middle one-third section of the forearm; the reference electrode was placed distal to the active electrode [9], and recordings were taken.

For abductor digiti minimi muscle (ADM), the active electrode was placed on the middle point between the distal elbow line and the 5th metacarpophalangeal joint; the reference electrode was placed distal to the active electrode in the metacarpophalangeal joint, and recordings were taken [10].

For PT, the active electrode was placed a distance of two fingers distal to the middle point between the humerus medial epicondyle and the biceps brachi muscle tendon; the reference electrode was placed distal to the active electrode, and recordings were taken [11].

For FCR, the active electrode was placed a distance of four fingers distal to the middle point between the humerus medial epicondyle and the biceps brachi muscle tendon; the reference electrode was placed distal to the active electrode, and recordings were taken [11].

For abductor pollicis brevis muscle (APB), the active electrode was placed on the middle point between the first metacarpophalangeal joint and the carpometacarpal joint on the palmar surface; the reference electrode was placed distal to the active electrode in the metacarpophalangeal joint, and recordings were taken [12]. 
Table 1. CMAP amplitudes of distal and proximal muscles

a CMAP amplitudes of muscles innervated by median or ulnar nerves $(n=60)$

\begin{tabular}{ll}
\hline Muscle & Mean amplitude $\pm \sigma, \mathrm{mV}$ \\
\hline FCU & $2.52 \pm 1.16$ \\
ADM & $5.81 \pm 3.13$ \\
PT & $2.90 \pm 1.20$ \\
APB & $6.88 \pm 2.77$ \\
FCR & $3.59 \pm 1.66$ \\
\hline
\end{tabular}

b Comparison of mean CMAP amplitudes of muscles innervated by the same nerves

\begin{tabular}{lcc}
\hline Comparison & $\mathrm{t}$ value & $\mathrm{p}$ value \\
\hline FCU with ADM & -8.96 & $<0.001$ \\
PT with APB & -11.25 & $<0.001$ \\
FCR with APB & -9.40 & $<0001$ \\
\hline
\end{tabular}

Table 2. CMAP durations of muscles innervated by median or ulnar nerves $(n=60)$

\begin{tabular}{ll}
\hline Muscle & $\begin{array}{l}\text { Mean CMAP } \\
\text { duration } \pm \sigma, \mathrm{ms}\end{array}$ \\
\hline FCU & $15.65 \pm 2.43$ \\
ADM & $13.55 \pm 1.78$ \\
PT & $16.38 \pm 2.39$ \\
APB & $13.40 \pm 1.79$ \\
FCR & $16.04 \pm 2.34$ \\
\hline
\end{tabular}

Study Scheme

Table 3. Nerve conduction velocities in the arm segment

a Nerve conduction velocities of muscles innervated by median or ulnar nerves $(n=60)$

\begin{tabular}{ll}
\hline Muscle & Mean velocity $\pm \sigma, \mathrm{m} / \mathrm{s}$ \\
\hline FCU & $78.27 \pm 6.55$ \\
ADM & $67.83 \pm 6.76$ \\
PT & $74.57 \pm 5.66$ \\
APB & $66.38 \pm 6.85$ \\
FCR & $74.23 \pm 5.88$ \\
\hline
\end{tabular}

b Comparison of nerve conduction velocities of median and ulnar nerves in the arm segment

\begin{tabular}{lcc}
\hline Comparison & t value & p value \\
\hline FCU with ADM & 12.12 & $<0.001$ \\
PT with APB & 12.14 & $<0.001$ \\
FCR with APB & 12.08 & $<0.001$ \\
\hline
\end{tabular}

For the recordings from each different muscle, we calculated the nerve conduction velocity, the CMAP amplitude and the response duration. Amplitude was measured as the interval between the negative and positive peaks at the highest and lowest wave (peak to peak); the latency was the distance from the stimulus artifact to the start of the first negative deflection and the response duration was measured from the start of the first negative deflection to the end of the $M$ response, i.e. the duration to the final return to the isoelectric line. The nerve conduction velocity using the distance between the proximal and distal stimuli of the ulnar and median nerves was calculated by an EMG device. CMAP amplitudes, response durations and velocities by calculating the values obtained from the proximal and distal stimulus for each muscle were compared. In the entire group enrolled in the trial, the CMAP amplitude and response duration values obtained from the proximal and distal stimuli from the same muscle were compared among themselves.

\section{Statistics}

To statistically assess the obtained data, Student's t test for paired groups and an independent-group t test were used to compare the male and female groups. Levene's test was also employed to assess whether the group variances were uniformly distributed.

\section{Results}

The mean age of the males and females in our cohort was $35.9 \pm 8.2$ and $35.5 \pm 9.0$ years, respectively. This difference in age was not statistically significant $(p>0.05)$.

Higher amplitude values were obtained in the distal muscles than in the proximal muscles innervated by the same nerve (ADM $5.81 \pm 3.13$ vs. FCU $2.52 \pm 1.16 \mathrm{mV}$, and APB $6.88 \pm 2.77$ vs. PT $2.90 \pm 1.20$ and FCR $3.59 \pm$ $1.66 \mathrm{mV} ; \mathrm{p}<0.001$ ) (table 1).

A comparison of the CMAP durations revealed longer response duration values for the proximal muscles than for the distal muscles innervated by the same nerve (FCU $15.65 \pm 2.43$ vs. $\mathrm{ADM} 13.55 \pm 1.78 \mathrm{~ms}$, and PT $16.38 \pm$ 2.39 and FCR $16.04 \pm 2.34$ vs. APB $13.40 \pm 1.79 \mathrm{~ms} ; \mathrm{p}<$ 0.001) (table 2). Compared to the distal muscles innervated by the same nerve, the nerve conduction velocity values obtained from the proximal muscles were higher (FCU $78.27 \pm 6.55$ vs. ADM $67.83 \pm 6.76 \mathrm{~m} / \mathrm{s}$, and PT $74.57 \pm 5.66$ and FCR $74.23 \pm 5.88$ vs. APB $66.38 \pm 6.85$ $\mathrm{m} / \mathrm{s} ; \mathrm{p}<0.001)$ (table 3$)$.

For all of the muscles assessed, the CMAP amplitude values from the proximal stimulus were significantly lower than the values obtained from the distal stimulus (FCU $2.16 \pm 1.10$ vs. $2.89 \pm 1.31 \mathrm{mV}$; ADM $5.14 \pm 3.18$ vs. 6.48 $\pm 3.25 \mathrm{mV}$; PT $2.55 \pm 1.07$ vs. $3.25 \pm 1.39 \mathrm{mV}$; FCR $3.32 \pm$ 1.71 vs. $3.85 \pm 1.76 \mathrm{mV}$; $\mathrm{APB} 5.90 \pm 2.72$ vs. $7.87 \pm 3.05$ $\mathrm{mV} ; \mathrm{p}<0.05)$; the CMAP response duration obtained via 
Table 4. Comparison of median and ulnar nerve conduction parameters

\begin{tabular}{lrcc}
\hline & Mean $\pm \sigma$ & \multicolumn{2}{l}{ Comparison } \\
\cline { 3 - 4 } & & t value & p value \\
\hline FCU-V & $78.27 \pm 6.55$ & 4.138 & $<0.001$ \\
PT-V & $74.57 \pm 5.66$ & & \\
FCU-V & $78.27 \pm 6.55$ & 4.651 & $<0.001$ \\
FCR-V & $74.23 \pm 5.88$ & & \\
FCU-A & $2.52 \pm 1.16$ & -2.277 & 0.026 \\
PT-A & $2.90 \pm 1.20$ & & \\
FCU-A & $2.52 \pm 1.16$ & -4.306 & $<0.001$ \\
FCR-A & $3.59 \pm 1.66$ & & \\
\hline
\end{tabular}

$\mathrm{V}=\operatorname{Velocity}(\mathrm{m} / \mathrm{s}) ; \mathrm{A}=$ amplitude $(\mathrm{mV})$.

Table 5. Comparison between male and female groups

\begin{tabular}{llllll}
\hline & \multicolumn{2}{l}{ Mean $\pm \sigma, \mathrm{ms}$} & & \multicolumn{2}{l}{ Comparison } \\
\cline { 2 - 3 } \cline { 6 - 7 } & male & female & & t value & p value \\
\hline FCU-D & $14.75 \pm 2.28$ & $16.55 \pm 2.27$ & & 3.06 & 0.003 \\
PT-D & $15.74 \pm 2.79$ & $17.02 \pm 1.72$ & & 2.13 & 0.037 \\
FCR-D & $15.33 \pm 2.46$ & $16.75 \pm 2.00$ & & 2.44 & 0.018 \\
\hline
\end{tabular}

$\mathrm{D}=$ Duration

the proximal stimulus was significantly longer than that obtained from the distal stimulus (FCU $16.27 \pm 2.54$ vs. $15.03 \pm 2.41 \mathrm{~ms} ; \mathrm{ADM} 14.05 \pm 1.95$ vs. $13.06 \pm 1.73 \mathrm{~ms}$; PT $17.05 \pm 2.51$ vs. $15.71 \pm 2.36 \mathrm{~ms}$; FCR $16.71 \pm 2.42$ vs. 15.38 $\pm 2.39 \mathrm{~ms} ;$ APB $13.90 \pm 1.70$ vs. $12.90 \pm 1.97 \mathrm{~ms} ; \mathrm{p}<0.05)$.

The recordings obtained from the proximal FCU stimulated by the ulnar nerve revealed significantly higher nerve conduction velocities and lower CMAP amplitude values than the values obtained from proximal PT and FCR (FCU $78.27 \pm 6.55$ vs. PT $74.57 \pm 5.66$ and FCR 74.23 $\pm 5.88 \mathrm{~m} / \mathrm{s}$, and FCU $2.52 \pm 1.16$ vs. PT $2.90 \pm 1.20$ and FCR $3.59 \pm 1.66 \mathrm{mV} ; \mathrm{p}<0.05)$. The CMAP response duration was shorter in the proximal muscle innervated by the ulnar nerve than that in the proximal muscle innervated by the median nerve (FCU $15.65 \pm 2.43$ vs. PT $16.38 \pm 2.39 \mathrm{~ms}$ ); however, there was no statistically significant difference $(p=0.223)$. A comparison of the recordings obtained from $\mathrm{ADM}$ and $\mathrm{APB}$ revealed no significant differences in terms of nerve conduction velocity (ADM $67.83 \pm 6.76$ vs. APB $66.38 \pm 6.85 \mathrm{~m} / \mathrm{s} ; \mathrm{p}>0.05$ ) and CMAP amplitudes (ADM $5.81 \pm 3.13$ vs. APB $6.88 \pm 2.77 \mathrm{mV}$; p > 0.05) (table 4).

Nerve Conduction Parameters in Proksimodistal Muscles
Comparing the mean CMAP amplitude values, the mean CMAP durations and the nerve conduction velocities of the male and female groups, the proximal muscles demonstrated significantly higher CMAP durations than the distal muscles innervated by the same nerve for females (FCU $14.75 \pm 2.28$ vs. $16.55 \pm 2.27 \mathrm{~ms}, \mathrm{p}=0.003$; PT 15.74 \pm 2.79 vs. $17.02 \pm 1.72 \mathrm{~ms}, \mathrm{p}=0.037$; FCR $15.33 \pm 2.46$ vs. $16.75 \pm 2.00 \mathrm{~ms}, \mathrm{p}=0.018$ ), but no statistically significant difference was detected in the other values (table 5).

\section{Discussion}

In this study, the proximal muscles innervated by the median or ulnar nerves consistently showed lower CMAP amplitude values, longer CMAP durations and higher conduction velocities than the distal muscles. Fibers with different diameters in the same nerve were noted.

In this study, the lower CMAP amplitude and longer durations obtained in the proximal muscles compared to the distal muscles innervated by the same nerve could be due to desynchronization associated with the high level of temporal dispersion resulting from the fibers having different nerve conduction velocities in the same nerve. When the amplitude of the action potential decreases, the area is reduced and the duration is longer in tandem with the distance between the stimulation and the recording point $[13,14]$. This is associated with temporal dispersion [15], which is a term that describes the desynchronization of the concomitantly stimulated components of the CMAP due to differences in conduction velocity. Therefore, temporal dispersion could explain the differences in CMAP amplitude, time and area between proximal and distal stimulations of the same nerve [16].

In this trial, the obtained CMAP amplitude and duration values from the proximal and distal stimuli in the same muscle revealed that the values of CMAP amplitude from the proximal stimulus were significantly lower than those from the distal stimulus. Furthermore, CMAP durations were significantly longer with the proximal stimulus than with the distal stimulus. The difference between the conduction rate of the slow fibers and the fast fibers in the peripheral nerve during conduction to a distant target became marked in relation to the short-distance conduction. Hence, the longer the conduction distance, the more did the stimuli in the slow fibers fall behind in relation to the fast fibers. Therefore, as the distance between the stimulation point and the recording site increases, the amplitude decreased and the time was prolonged even if the same number of fibers contributed to 
the action potential. In the trial by Olney and Miller [17], the authors demonstrated a linear correlation between the response size and the length of the nerve segment. Mattler et al. [18] noted that the proximodistal CMAP changes increased with the length of the nerve segment. The data obtained in our study were similar to the data obtained in these two studies. This could be a confirmation of the presence of the nerve fibers with different velocities in the same nerve.

In this study, ulnar nerve conduction parameters were also performed by stimulating from different sites and recording from the same muscle. The values of CMAP amplitude from the proximal stimulus were significantly lower than those from the distal stimulus. In another trial with ulnar nerves, Oğuzhanoğlu et al. [3] detected that the arm segment amplitude values were lower than the forearm segment amplitude values in the hypothenar region recordings, and authors attributed this findings to temporal dispersion. This study showed that the same findings also apply to the median nerve in addition to ulnar nerve findings. The mechanism proposed for a reduction in the proximal CMAP area and the amplitude values is phase cancellation that occurs during the composite of the temporally diffused diphasic and polyphasic action potentials [19]. Phase cancellation occurs during the algebraic sum of the single muscle fiber action potentials constituting the motor unit action potential. However, phase cancellation related to the motor unit action potential is independent of the site of stimulus. Even so, we can state that CMAP formed by the sum of different motor unit action potentials is associated with temporal dispersion [17].

Motor and sensorial peripheral nerves consist of nerve fibers with different nerve conduction rates. The difference in the nerve conduction rate is said to be within a wide range such as between 12 and $13 \mathrm{~m} / \mathrm{s}$ [20]. Considering that the fastest conducting A-alpha fibers have a rate around $60 \mathrm{~m} / \mathrm{s}$, this difference matters. This variation in nerve conduction velocities results in a desynchronized activation in those obtained via proximal stimulus more prominently than the motor unit action potentials obtained via distal stimulation. In our study, longer durations were obtained in the proximal muscles compared to the distal muscles innervated by the same nerve, and also the CMAP durations were significantly longer with proximal stimulus than with distal stimulus. In the case of proximal stimulation, i.e. along the longer conduction path, the difference between the fast and slow conducting fibers becomes more marked compared to the stimulus from the distal muscle along the shorter conduction path. This explains the longer CMAP duration obtained by proximal stimulation compared to that obtained by distal stimulus. In addition, desynchronized activation of the motor unit action potentials results in further increased phase cancellation in the terminal section of the CMAP-negative phase.

Temporal dispersion may increase in cases of peripheral nerve injury; the effect of temporal dispersion on CMAP amplitude and duration was demonstrated in subjects with normal nerves and subjects with peripheral neuropathy [21]. Abnormal desynchronization between nerve fibers in focal demyelinated regions may result in the negative and positive phases of the diphasic action potential constituting the motor response canceling each other. This cancellation increases the temporal dispersion by reducing the amplitude. In the case of demyelinating neuropathies, the CMAP amplitude by distal stimulus decreases mildly or moderately due to abnormal temporal dispersion. Phase cancellation and distal latency can be delayed due to demyelination. With additional proximal stimulus, the CMAP amplitude decreases due to temporal dispersion as well as due to conduction block in some fibers. Since the likelihood of the nerve action potentials crossing through the demyelinated segments is increased, it is reduced markedly [22]. This study showed that the proximal muscles innervated by the median or ulnar nerves had lower CMAP amplitude values, longer CMAP durations and higher conduction velocities than distal muscles. In this context, the trial is important since it paves the way for a better understanding of physiological changes in nerve conduction parameters obtained from proximal and distal muscles in the same nerve segment and neuropathic conditions.

In this trial, no significant differences in nerve conduction rates and CMAP amplitudes between the proximal and distal muscles were detected between males and females. However, different results have been obtained in other studies. Alemdar [23] compared the sensory responses in the median and ulnar nerves and detected a significantly higher mean sensory nerve action potential amplitude and a shorter distal latency in females compared to males. Bolton and Carter [24] similarly detected higher sensory action potential amplitude values in median and ulnar nerves in females. Hennessey et al. [25] compared the nerve conduction rates, distal latencies and amplitude values between males and females. These authors reported a higher sensory action potential amplitude value only in females.

While there have been a few studies [25-27] explaining the gender effect on nerve conduction with the fact that males have more manual professions and their ulnar nerve is thus more susceptible to injury, it seems difficult to generalize this inference. The higher sensory nerve action potential amplitudes in females, reported in some 
studies, may be attributed to the thinner cutaneous and subcutaneous tissue of female fingers and the fact that the action potential is recorded on a short distance. However, previous studies did not report consistent results for the differences in nerve conduction parameters between genders. This fact suggests the potential presence of factors that have not yet been assessed or measured, and additional trials are clearly necessary.

\section{Conclusion}

In this study, proximal muscles innervated by median or ulnar nerves had lower CMAP amplitude values, longer CMAP durations and higher conduction velocities than distal muscles innervated by median or ulnar nerves. The fast- and slow-conducting fibers progressed together in the same nerve, and desynchronization was associated with a high level of temporal dispersion resulting from the fibers of different nerve conduction rates.

\section{Acknowledgement}

This study was supported by the Pamukkale University Scientific Research Projects Coordination Unit (No. 2013TPF018).

\section{Disclosure Statement}

The authors report no conflicts of interest.

\section{References}

1 Henneman E, Olson CB: Relations between structure and function in the design of skeletal muscles. J Neurophysiol 1965;28:581-598

2 Cullheim S: Relations between cell body size, axon diameter and axon conduction velocity of cat sciatic alfa motoneurons stained with horseradish peroxidase. Neurosci Lett 1978;8: $17-20$.

-3 Oğuzhanoğlu A, Güler S, Cam M, et al: Conduction in ulnar nerve bundles that innervate the proximal and distal muscles: a clinical trial. BMC Neurol 2010;10:81.

-4 Oğuzhanoğlu A, Erdoğan Ç, Tabak E, et al: Comparison of conduction velocities of nerve fibers to smaller and larger muscles in rats. Int J Neurosci 2010;120:76-79.

5 Buchtal F, Schmalbruch H: Motor unit of mammalian muscle. Physiol Rev 1980;60:90142.

6 Gassel MM, Trojaborg W: Clinical and electrophysiological study of the pattern of conduction times in the distribution of the sciatic nerve. J Neurol Neurosurg Psychiatry 1964; 27:351-357.

7 Erdogan Ç, Cenikli U, Değirmenci E, et al: Effect of hyperglycemia on conduction parameters of tibial nerve's fibers to different muscles: a rat model. J Neurosci Rural Pract 2013; $4: 9-12$.

8 Werner RA: Electrodiagnostic evaluation of carpal tunnel syndrome and ulnar neuropathies. PM R 2013;5:14-21.

-9 Felsenthal G, Brockman PS, Mondell DL, et al: Proximal forearm ulnar nerve conduction techniques. Arch Phys Med Rehabil 1986;67: 440-444.
10 Azma K, Bahmanteimoury K, Tavana B, et al: Two measurement methods of motor ulnar nerve conduction velocity at the elbow: a comparative study. Neurol India 2007;55: 145-147.

11 Delagi EF, Perotto A: Anatomic Guide for the Electromyographer, ed 2. Springfield, Charles C. Thomas, 1980, pp 4, 6, 46, 48, 60.

12 Jablecki CK, Andary MT, So YT, et al: Literature review of the usefulness of nerve conduction studies and electromyography for the evaluation of patients with carpal tunnel syndrome. AAEM Quality Assurance Committee. Muscle Nerve 1993;16:1392-1414.

13 Preston DE, Shapiro BE: Polyneuropathy; in Preston DE, Shapiro BE: Electromyography and Neuromuscular Disorders: Clinical-Electrophysiologic Correlations. Boston, Butterworth-Heinemann, 1998, pp 357-370.

14 Johnsen B, Fuglsang-Frederiksen A, de Carvalho $\mathrm{M}$, et al: Amplitude, area and duration of the compound muscle action potential change in different ways over the length of the ulnar nerve. Clin Neurophysiol 2006;117: 2085-2092.

15 Kimura J, Sakimura Y, Machida M, et al: Effect of desynchronized inputs on compound sensory and muscle action potentials. Muscle Nerve 1988;11:694-702.

16 Kimura J, Machida M, Ishida T, et al: Relation between size of compound sensory or muscle action potentials and length of nerve segment. Neurology 1986;36:647-652.

17 Olney RK, Miller RG: Conduction block in compression neuropathy: recognition and quantification. Muscle Nerve 1984;7:662667.
18 Mattler WJS, Müller T, Georgiadis D, et al: Length dependence of variables associated with temporal dispersion in human motor nerves. Muscle Nerve 2001;24:527-533.

19 Kimura J: Principles and pitfalls of nerve conduction studies. Ann Neurol 1984;16:415-429.

20 Dorfman LJ: The distribution of conduction velocities (DCV) in peripheral nerves: a review. Muscle Nerve 1984;7:2-11.

21 Gilliatt RW, Sears TA: Sensory nerve action potentials in patients with peripheral nerve lesions. J Neurol Neurosurg Psychiatry 1958; 21:109-118.

22 Raynor EM, Ross MH, Shefner JM, et al: Differentiation between axonal and demyelinating neuropathies: identical segments recorded from proximal and distal muscles. Muscle Nerve 1995; 18:402-408.

23 Alemdar M: Effects of gender and age on median and ulnar nerve sensory responses over ring finger. J Electromyogr Kinesiol 2014;24: 52-57.

24 Bolton CF, Carter KM: Human sensory nerve compound action potential amplitude: variation with sex and circumference. J Neurol Neurosurg Psychiatry 1980;43:925-928.

25 Hennessey WJ, Falco FJ, Goldberg G, et al: Gender and arm length: influence on nerve conduction parameters in the upper limb. Arch Phys Med Rehabil 1994;75:265-269.

26 Stetson DS, Albers JW, Silverstein BA, et al: Effects of age, sex, and anthropometric factors on nerve conduction measures. Muscle Nerve 1992;15:1095-1104.

27 Kommalage M, Gunawardena S: Influence of age, gender and sidedness on ulnar nerve conduction. J Clin Neurophysiol 2013;30:98-101. 